\title{
Effects of layer eccentricity on the super-resonant states of active cylindrical core-shell nano-particles
}

\author{
Rasmus Ø. Thorsen and Samel Arslanagić* \\ Department of Electrical Engineering, Technical University of Denmark, Ørsteds Plads, Bldg. 348, 2800 Kongens Lyngby, Denmark
}

Received 15 September 2015 / Accepted 8 November 2015

\begin{abstract}
This work reports on the effects of layer eccentricity on the resonant properties of active cylindrical coreshell nano-particles excited by a near-by exterior magnetic line source. The core-shell particles consist of a silver core layered with a silica shell. For a fixed over-all radius of the nano-particle equal to $30 \mathrm{~nm}$, we investigate designs with relatively small (radius equal to $6 \mathrm{~nm}$ ) and large (radius equal to $24 \mathrm{~nm}$ ) silver cores and we quantify their performance characteristics in terms of the near- and far-field properties. Our results show that the super-resonances, known to exist in the concentric version of these nano-particles, are significantly influenced by introducing eccentricity (through displacements of the silver core relative to the silica shell). In particular, their amplitude responses are found to diminish significantly for silver core displacements $\geq 3 \mathrm{~nm}$ for the small core case, and even for displacements $\geq 1 \mathrm{~nm}$ for the large core case. The present results are useful from the experimental point of view since slight displacements of the centers of the core and shell parts of the investigated nano-particles are likely to occur in standard fabrication processes.
\end{abstract}

Key words: Plasmonics, Nano-particles, Scattering, Gain, Eccentricity.

\section{Introduction}

Recent years have witnessed a truly increased interest in passive and active nano-particle (NP) systems, and the fascinating plethora of their applications within the emerging areas of nano-photonics including nano-sensors and nano-antennas, lasers and spasers, as well as circuits and artificial material design at optical frequencies [1-9]. A very successful outcome of this research field was the demonstration that specific electrically small and active coated NPs, based on plasmonic and dielectric materials, may possess highly resonant properties making them useful for many of the above mentioned applications, particularly for the design of efficient nano-sensors, antennas and amplifiers. This is because of the enhanced light-matter interactions in these active NPs which physically are due to the surface plasmon resonances occurring at the interface between the plasmonic and dielectric parts of the NP.

Over the years, a great variety of NP shapes was investigated [1]. However, the most attention was devoted to the spherical designs and to some extent to the circular cylindrical designs as well. Spherical active coated NPs, consisting of a dielectric core impregnated with a canonical, constant frequency gain medium, layered with a plasmonic shell, were

*e-mail: sar@elektro.dtu.dk studied for potential nano-laser and directivity enhancement applications in $[6,10,11]$, as well as for potential nano-sensing applications [12]. Moreover, similar NPs were employed in the design of optical metamaterials [8], whereas artificial material designs utilizing more realistic spherical active NPs were reported in [9]. The fundamental properties of truncated cylindrical active NPs (nano-rods) were examined in e.g., the works of $[13,14]$. While most of the efforts were concentrated on the use of NPs under the plane wave applications, the effects of their excitation by localized radiation sources were examined recently in [15-17] for spherical as well as cylindrical coated NP configurations. This effectively allows one to study, e.g., the effects of the resonant coated NPs on the fluorescence of the near-by molecules which can be modelled as Hertzian dipole antennas. In particular, super-resonant states have been identified in $[16,17]$ in active coated NPs. At these superresonant states, the plasmonic losses in the NP are fully overcome, and thus a very large enhancement of the power radiated by the near-by localized source is obtained.

The great majority of the works mentioned above in the area of active and passive coated NPs have examined the properties of the so-called concentric coated NPs, i.e., the centers of the two layers comprising the NP are coincident. However, from the experimental point of view, slight displacements of the centers of the different NP parts are likely to occur in 
standard fabrication processes. As a consequence, it is relevant to examine how such displacements will influence the superresonant states known to exist in the concentric active NPs.

The purpose of the present work is to extend the study in [17] of cylindrical active and concentric NPs to account for the influence of the eccentricity in such particles on their super-resonant states. The eccentricity will be introduced by appropriate displacements of the core center, relative to the center of the surrounding shell. The core will be displaced along the various directions, by an amount of 1,3 , and $5 \mathrm{~nm}$. The displacement of $1 \mathrm{~nm}$ is more likely to occur in a standard fabrication process then the 3 and $5 \mathrm{~nm}$ displacements. The latter would be displacements one introduces deliberately in the design. Nevertheless, for the sake of completeness and the systematic nature of the study of these effects, these larger displacements are also included in the present work. In all cases, the active NP configurations consist of a plasmonic (silver) core, covered by a dielectric (silica) shell, being excited by a near-by magnetic line source. The configuration is analyzed analytically employing the eigenfunction expansion technique in conjunction with the addition theorem for cylindrical wave functions and boundary conditions for the determination of the fields in various regions [18]. The developed analytical results will be implemented in Matlab, and further verified numerically though comparisons with the results obtained in Comsol Multiphysics. For a fixed overall size of the NP of radius equal to $30 \mathrm{~nm}$, designs with small (radius of $6 \mathrm{~nm}$ ) and large (radius of $24 \mathrm{~nm}$ ) cores are analyzed in terms of their near- and far-fields, total power spectrum, as well as the power flow properties. Our results show that the super-resonances in the concentric NPs are significantly influenced by displacements of the silver core relative to the silica shell, this being more the case for the large-core NP. In particular, the amplitude of these resonances is found to diminish significantly for silver core displacements $\geq 3 \mathrm{~nm}$ for the small core case, and even for displacements $\geq 1 \mathrm{~nm}$ for the large core case; in all cases, the decrease of the strength of the excited dominant mode is more profound for the large-core NP. We note that the present manuscript is a heavily extended version of a recent conference contribution [19]. In the present paper, a detailed account on the analytical solution and model are included, as are additional results for core displacements along the various directions accompanied with power spectrum and power flow density interpretations. In addition, it is useful to note that similar effects of core displacements were investigated recently for a more complicated three-layer NP where a few additional features, not present for the currently investigated two-layer NPs, were found to occur [20].

This paper is organized as follows. Section 2 presents the configuration of the eccentrically layered NP and outlines briefly the analytical method of its analysis. This section also summarized the specific material and geometric details related to the investigated configurations. Section 3 presents and discusses all of the obtained results. Finally, Section 4 includes the summary and conclusions of the work. Two appendixes are included in the present work. Appendix A includes a detailed account on the analytical solution of the problem at hand, and Appendix B includes a comparison between the analytical and numerical results that confirms the former.

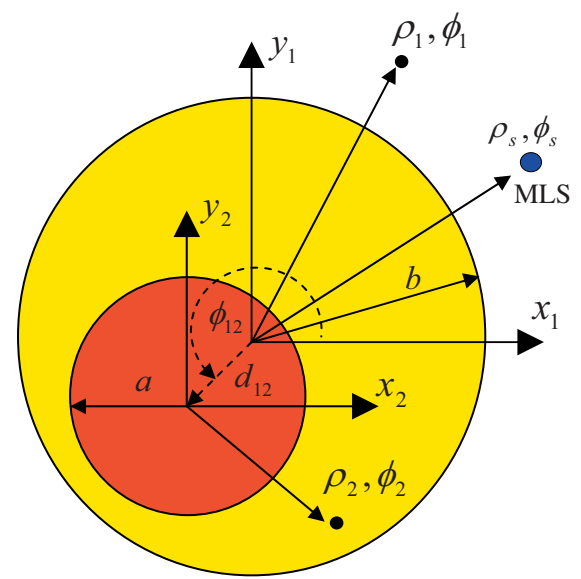

Figure 1. The configuration of an eccentrically layered active coreshell nano-particle excited by a near-by exterior MLS.

Throughout this work, the time-factor $\exp (j \omega t)$, with $\omega$ being the angular frequency and $t$ being the time, is assumed and suppressed.

\section{Configuration and methods of analysis}

The nano-particle (NP) configuration of the present interest consists on an inner plasmonic core (region 1), of radius $a$, coated eccentrically by a dielectric shell, region 2 , of outer radius $b$, see Figure 1 . The NP is located in free-space (region 3). The permittivity, permeability, and wavenumber of regions 1 and 2 are denoted by $\varepsilon_{l}, \mu_{l}$, and $k_{l}=\omega \sqrt{\varepsilon_{l} \mu_{l}}$, respectively, with $l=1,2$, whereas the corresponding parameters of region 3 are denoted by $\varepsilon_{0}, \mu_{0}$, and $k_{0}=\omega \sqrt{\varepsilon_{0} \mu_{0}}=2 \pi / \lambda$, where $\lambda$ is the wavelength. The NP is excited by an infinitely long magnetic line source (MLS) of current with magnitude $I_{\mathrm{m}}[\mathrm{V}]$ located outside of the NP. Two Cartesian $\left(x_{i}, y_{i}, z_{i}\right)$, and the associated cylindrical $\left(\rho_{i}\right.$, $\phi_{i}, z_{i}$ ), coordinate systems $i=1,2$ are introduced so that the origin of the $i=1(i=2)$ coordinate system coincides with the center of the dielectric shell (plasmonic core), and such that the $z_{i}$-axes coincide with the respective axes of the NP. The coordinates of the MLS are $\left(\rho_{s}, \phi_{s}\right)$, while those of the observation point are $\left(\rho_{1}, \phi_{1}\right)$ outside the NP, and $\left(\rho_{2}, \phi_{2}\right)$ inside the NP. The eccentricity (the displacement of the plasmonic core from the center of the dielectric shell) is described by the radial distance $d_{12}$ from the core and shell centers, and its angle $\phi_{12}$ with respect to the $x_{1}$-axis, see Figure 1 .

In this work, an analytical solution is obtained for the problem illustrated in Figure 1. For the moment, we merely outline the main steps of this solution, and refer to Appendix A for the details of the solution. The solution is based on the eigenfunction expansion technique, and we adopt the approach outlined in [18]. The fields of the MLS, as well as the unknown fields due to the NP, are expanded in terms of cylindrical waves. Appropriate addition theorems for the radial functions are used to express the fields pertaining to a specific interface in the same coordinate system. This is required for the subsequent application of the boundary conditions, which then allows for the determination of the unknown expansion coefficients 
Table 1. Summary of material and geometric parameters of the two NP configurations.

\begin{tabular}{cccccccc}
\hline Design & Core & Shell & Host & $b[\mathrm{~nm}]$ & $a[\mathrm{~nm}]$ & $I_{\mathrm{m}}[\mathrm{V}]$ & $\rho_{s}, \phi_{s}\left[\mathrm{~nm},{ }^{\circ}\right]$ \\
\hline Small-core & $\mathrm{Ag}$ & $\mathrm{SiO}_{2}$ & $\varepsilon_{0}, \mu_{0}$ & 30 & 6 & 1 & 35,0 \\
Large-core & $\mathrm{Ag}$ & $\mathrm{SiO}_{2}$ & $\varepsilon_{0}, \mu_{0}$ & 30 & 24 & 1 & 35,0 \\
\hline
\end{tabular}

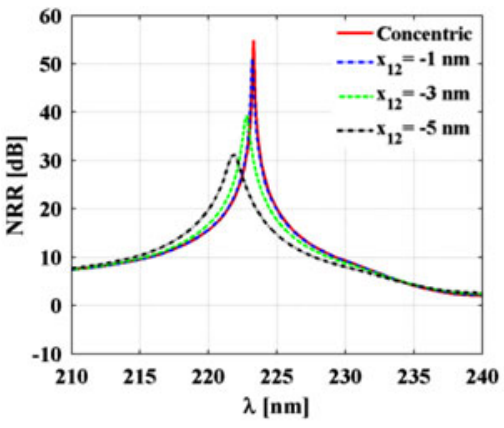

(a)

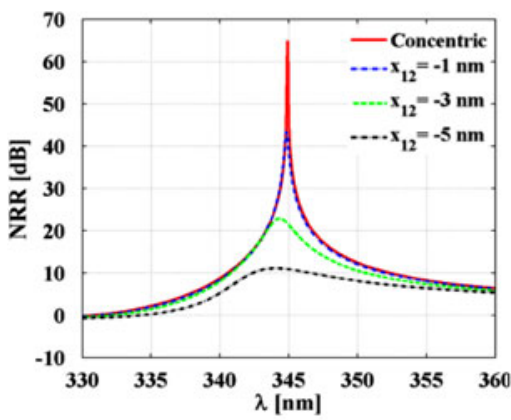

(d)

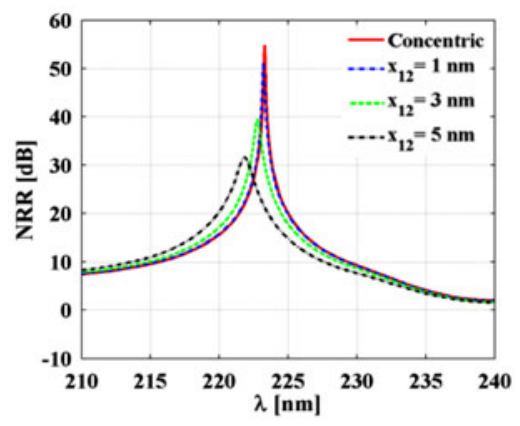

(b)

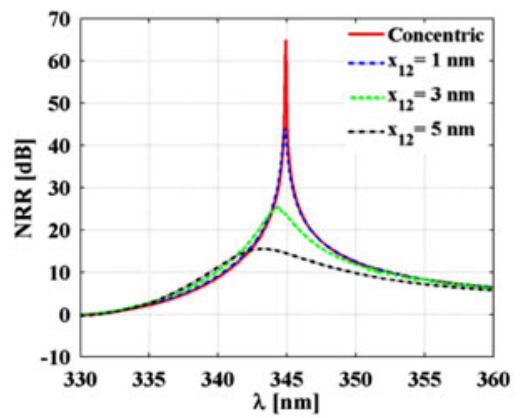

(e)

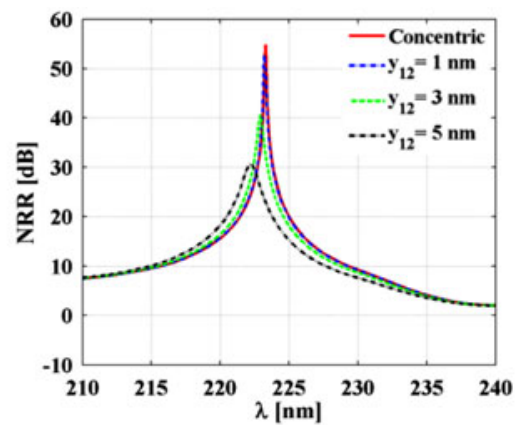

(c)

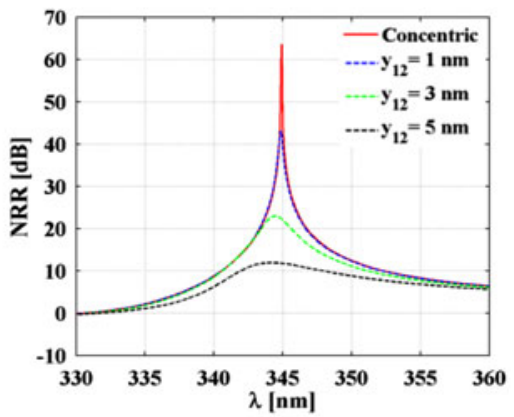

(f)

Figure 2. NRR as a function of the wavelength $\lambda$ for the small-core NP (a)-(c), and the large-core NP (d)-(f) as a function of the eccentricity along $\pm x$ - and $y$-directions indicated in the legends of the respective figures. The result for the reference, concentric NP is included in all cases. See the main text for further explanations.

of the NP fields. Once the coefficients have been determined, the fields are known at all points of space; and they are used to determine a number of other quantities to quantify the performance of the investigated NP. These derived quantities will be introduced in the later sections of this work.

As to the specific NP configurations investigated here, we focus our attention on two NPs, each made of a silver (Ag) core and a silica $\left(\mathrm{SiO}_{2}\right)$ shell. For the first design, referred to as the small-core design, we set $a=6 \mathrm{~nm}, b=30 \mathrm{~nm}$, while for the second design, referred to as the large-core design, we set $a=24 \mathrm{~nm}, b=30 \mathrm{~nm}$. Owing to the nano-scale size of the plasmonic core, the size-dependency effects of the silver permittivity have been taken into account [6]. In order to simulate an active NP, the gain is introduced in the silica shell region via its permittivity $\varepsilon_{2}=\varepsilon_{0}(n-j \kappa)$ where $n=\sqrt{2.05}$ is the refractive index of silica at the considered wavelengths, while the parameter $\kappa<0$ represents the gain; a value of $\kappa$ is to be found for which the silver core losses are overcome, and the super-resonant states of the NP are identified.

In our investigations, attention is devoted to the influence of the eccentricity achieved through the core displacements relative to the surrounding shell. To this end, the eccentricity parameters $d_{12}$ and $\phi_{12}$ are varied. In particular, the core displacements will be considered along the positive and negative $x$-axis (designated as $x_{12}= \pm d$ nm with $x$ being the amount of displacement) and along the positive $y$-axis (designated as $y_{12}=+d \mathrm{~nm}$ ). In all cases, the MLS will be driven by a current of magnitude $I_{\mathrm{m}}=1 \mathrm{~V}$, and it will be located on the positive $x$-axis at $\rho_{s}=35 \mathrm{~nm}$. A summary of the relevant parameters of the investigated NPs is found in Table 1.

\section{Results and discussion}

For the active concentric NPs, a super-resonant state, where the NRR is maximized, occurs for $\kappa=-1.271$ at $\lambda \approx 223 \mathrm{~nm}$ (the small-core NP), and for $\kappa=-1.033$ at $\lambda \approx 345 \mathrm{~nm}$ (the large-core NP). The resulting NRRs for these two concentric cases are around $55 \mathrm{~dB}$ for the small-core NP, and around $65 \mathrm{~dB}$ for the large-core NP. To investigate the influence of the eccentricity, we next displace the core by an amount: $x_{12}= \pm(1,3,5) \mathrm{nm}$, and $y_{12}=+(1,3,5) \mathrm{nm}$. Figures $2 \mathrm{a}-2 \mathrm{c}$, show the NRR as a function of the wavelength $\lambda$ for the small-core NP, whereas Figures $2 \mathrm{~d}-2 \mathrm{f}$ show the corresponding results for the large-core NP. The core displacements are indicated in the legends of the respective figures. In each of the 


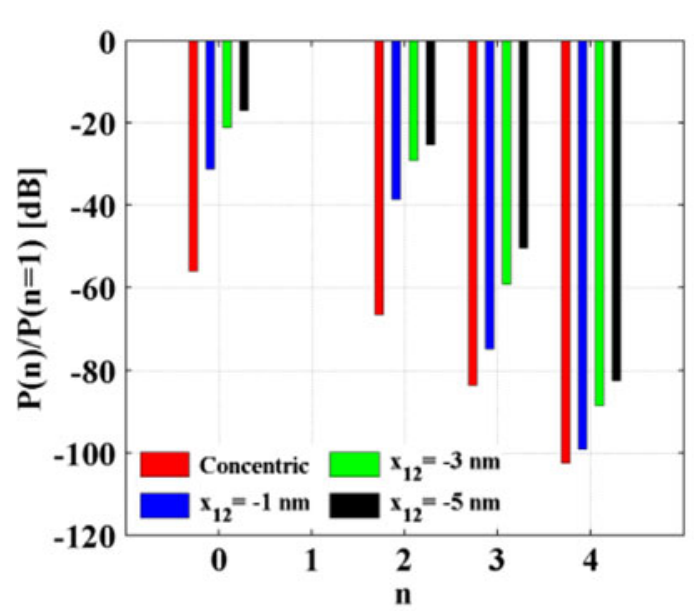

(a)

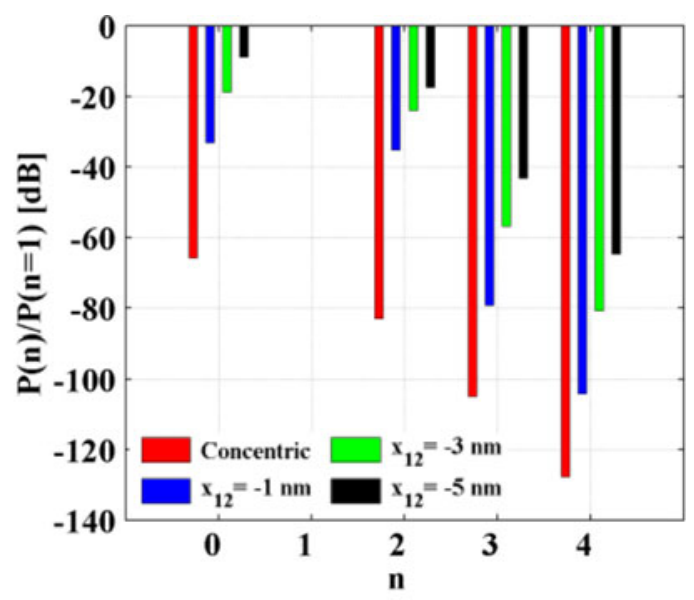

(b)

Figure 3. The total power spectrum of the $n$-th mode, i.e., the total power in the $n=0,1,2,3$ and 4 modes, normalized by the total power in the dipole $(n=1)$ mode. The results in (a) apply for the small-core NP, and the results in (b) apply for the large-core NP. The spectrum is shown for the concentric as well as eccentric cases; for the latter, the core displacements along the negative $x$-axis, $x_{12}=-1,3$, and $5 \mathrm{~nm}$, are considered.

figures, the results for the corresponding concentric NPs are included for reference purposes. Regardless of the core size, the core displacements introduce a very slight shift in the resonance towards the shorter wavelengths. More notably, however, the core displacements reduce the NRR values as compared to the values obtained in the concentric cases. This reduction is significantly more profound for the large-core NP than for the small-core NP. Specifically, the NRR experiences a significant drop (around $20 \mathrm{~dB}$ ) for the large-core NP even for displacements of $1 \mathrm{~nm}$, whereas the corresponding drop for the small-core NP is around $4 \mathrm{~dB}$. Significant decrease of the NRR for the presently investigated small-core NP is in evidence only for core displacements $\geq 3 \mathrm{~nm}$, while the large-core NP is affected, even more significantly, already for displacements $\geq 1 \mathrm{~nm}$. Moreover, whereas very similar results are obtained irrespective of the core displacement direction for the small-core NP (a)-(c), some variation in the NRR is found for the large-core NP depending on the core displacement direction, as evidenced by the results in Figures $2 \mathrm{~d}$ and 2e.

The behavior of the NRR in Figure 2 is next further examined through the considerations of the so-called normalized total power spectrum of the $n$ 'th mode. This quantity is the total power in the $n=0,1,2,3$, and 4 mode, normalized by the total power contained in the dipole $(n=1)$ mode. These results are shown in Figure $3 \mathrm{a}$ for the small-core NP, and in Figure $3 b$ for the large-core NP. The results for the concentric as well as eccentric NPs, with core displacements of $x_{12}=-(1,3,5) \mathrm{nm}$, are shown in the figures. In both concentric NP cases (red bars in Figure 3), it is clear that the dipole mode dominates the response of the NP. Thus the large corresponding values of the NRR reported in Figure 2 are due to the excitation of this strong dipole mode inside the respective NPs. The dipole mode dominance is somewhat more emphasized in the large-core NP than in the small-core NP. As the core gets displaced by $x_{12}=-1 \mathrm{~nm}$ (blue bars), $x_{12}=-3 \mathrm{~nm}$ (green bars), and $x_{12}=-5 \mathrm{~nm}$ (black bars), one finds that other modes start contributing more notably, although the dipole mode still dominates the response. The appearance of these additional modes with the increasing core displacements is more notable for the large-core NP than for the small-core NP. To illustrate, whereas the $n=0$ (monopole) and $n=2$ (quadrupole) are down by more than $60 \mathrm{~dB}$ and $80 \mathrm{~dB}$, respectively, relative to the dipole mode in the concentric large-core $\mathrm{NP}$, they are down by less than $10 \mathrm{~dB}$ and $20 \mathrm{~dB}$, respectively, for the eccentric, large-core NP with $x_{12}=-5 \mathrm{~nm}$, see Figure $3 \mathrm{~b}$. The relative increase of the strength of these modes in the corresponding eccentric small-core NP is not as profound as can be clearly seen in Figure $3 \mathrm{a}$. We note that similar results to those reported in Figure 3 are obtained for the core displacements along the positive $x$ - and $y$ - axes, and are therefore not included here.

The fact that the NRR peaks in the concentric cases in Figure 2 is due to a strong dipole mode is further confirmed by the results in Figure 4, which shows the power flow density $\boldsymbol{S}=\operatorname{Re}\left\{\boldsymbol{E} \times \boldsymbol{H}^{*}\right\} / 2$, with $\boldsymbol{E}(\boldsymbol{H})$ being the total electric (magnetic) field, and * (asterisk) denoting the complex conjugate. More specifically, the magnitude of the power flow density, $\operatorname{lol}_{10}|\boldsymbol{S}|$, (with $|\boldsymbol{S}|$ normalized by $1 \mathrm{~W} / \mathrm{m}^{2}$ prior to taking the logarithm) is shown in color, along with the direction of $\boldsymbol{S}$, which is shown by arrows. Figures $4 \mathrm{a}-4 \mathrm{c}$ apply for the smallcore NP, while Figures 4d-4f apply for the large-core NP. The concentric cases are shown in Figures $4 \mathrm{a}$ and $4 \mathrm{~d}$, while the core displacement results for $x_{12}=5 \mathrm{~nm}$ are shown in Figures $4 \mathrm{~b}$ and $4 \mathrm{e}$, and those for the $x_{12}=-5 \mathrm{~nm}$ case are shown in Figures $4 \mathrm{c}$ and $4 \mathrm{f}$. The concentric cases show a clear and symmetric dipole power flow pattern with the magnitude of the response outside of the NP being notably larger for the large-core case. This is in perfect agreement with the larger NRR values obtained for this NP $(65 \mathrm{~dB})$ as compared to the small-core NP $(55 \mathrm{~dB})$. Note, however, that the power flow density inside the small-core NP is rather large in the active silica shell region close to the part of the surface of the Ag core that crosses the $y$-axis. As the eccentricity is introduced, the dominant mode is still a dipole mode, although it is rather 


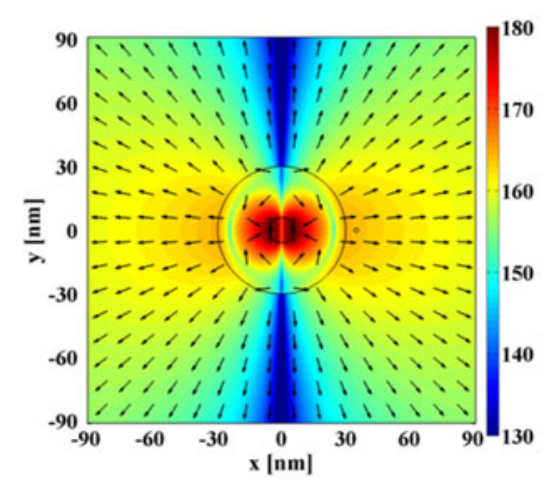

(a)

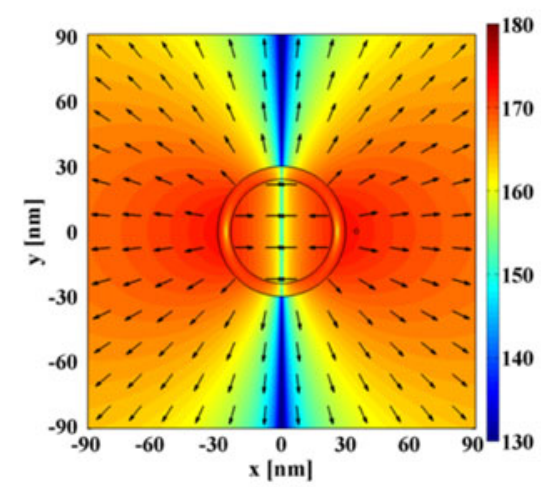

(d)

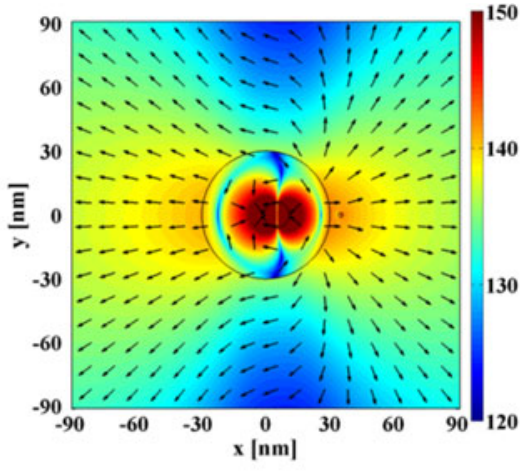

(b)

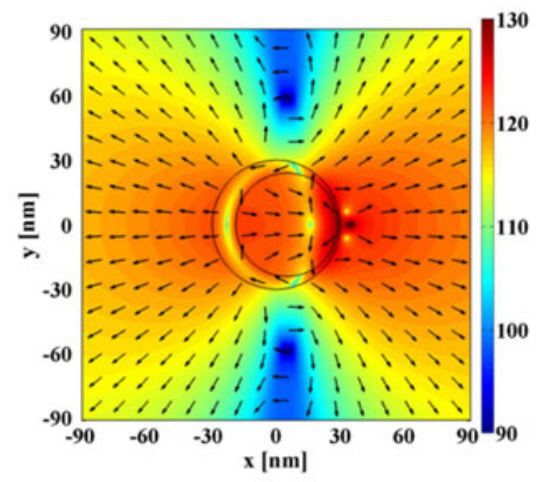

(e)

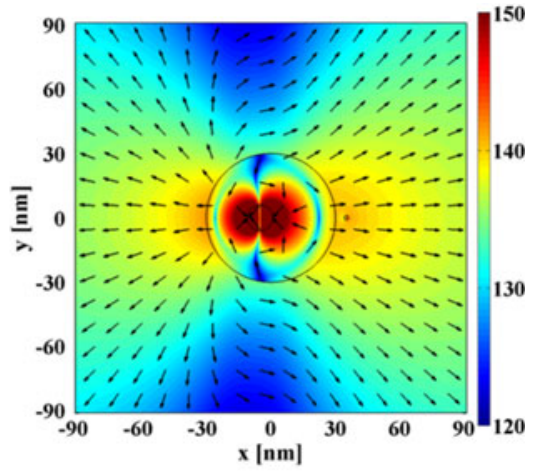

(c)

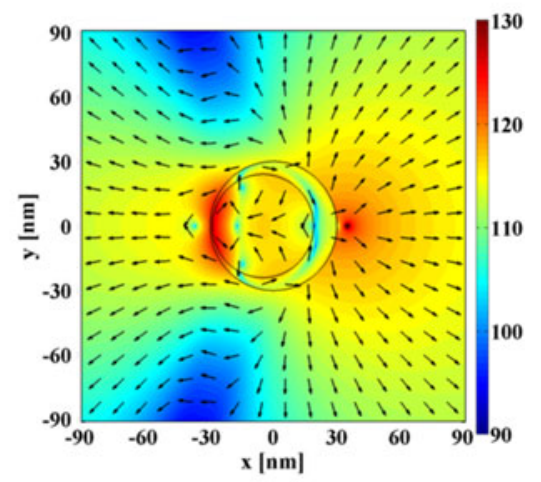

(f)

Figure 4. The magnitude of the power flow density, $10 \log _{10}|S|$, (color), and its direction (arrows) for small-core NPs (a)-(c), and large-core NPs (d)-(f). The power flow density is shown for the concentric, (a) and (d), as well as eccentric, (b)-(c) and (e)-(f), cases. For the latter, the core displacements of $x_{12}=5 \mathrm{~nm}$, are considered in (b) and (e), whereas $x_{12}=-5 \mathrm{~nm}$ in (c) and (f). The results are shown in a square region of $90 \times 90 \mathrm{~nm}^{2}$. Curves illustrating the cylindrical surfaces of the NP are also shown.

asymmetric and is significantly decreased in strength. This is the reason for the reduced NRR values reported in Figure 2 for the eccentric NPs. It is also interesting to note how the power flow density inside the two NPs is very different from one another. In particular, apart from the overall dipolar response, as seen from the outside in both cases, the power flow for the small-core NP shows an additional dipole mode inside the NP not found for the large-core NP. The rather small size of the Ag core makes it radiate as a dipole on its own. However, it is tightly bound to the surface of the core; and, therefore, it does not interact efficiently with the large active silica shell. This is also one of the reasons for the overall diminished responses in the small-core cases relative to the large-core ones.

Our results have shown that the resonant modes excited in a concentric NP of a fixed overall size, but with relatively small and large silver cores covered with active silica shells, are significantly affected as the core gets slightly displaced. The influence was most notable for the large-core NPs, for which significant deterioration of the super-resonant states was observed for core displacements as small as $1 \mathrm{~nm}$.

We note conclusively that each of the eccentric cases studied above can be optimized by small adjustments of the gain parameter $\kappa$ in order to more effectively excite their superresonant states and achieve much larger enhancements of their NRR values than in the above reported eccentric cases.
Compared to the optimized concentric NPs, the magnitude of $\kappa$ required to optimize the eccentric cases with the largest core displacement was found to increase for all large-core NPs, and for the vast majority of the investigated small-core NPs.

\section{Summary and conclusions}

In this work, the influence of the layer eccentricity on the performance of active cylindrical nano-particles excited by a near-by magnetic line source was investigated. The nano-particles consisted of a silver core layered with an active silica shell. Our investigation was devoted to two specific nanoparticle geometries. Their overall radius was fixed to $30 \mathrm{~nm}$, while the silver core radius was set to $6 \mathrm{~nm}$, for the small-core nano-particle, and $24 \mathrm{~nm}$, for the large-core nano-particle. The gain medium was simulated with a canonical, constant frequency, gain model. The investigation was analytical in nature, and was further confirmed by the numerical results as obtained in Comsol Multiphysics. The quantities used to assess the performance of the investigated nano-particles were the so-called normalized radiation resistance, total radiated power spectrum, near-field distributions as well as the distributions of the power flow density inside and outside the nanoparticles. 
Our analysis has shown that the super-resonances, i.e., the large values of the normalized radiation resistance, known to exist in the concentric version of the investigated nano-particles, are significantly influenced by slight displacements of the silver core. This was found to be most pronounced with the studied large-core nano-particles, for which the amplitude responses of the super-resonant states were found to diminish significantly for core displacements even as small as $1 \mathrm{~nm}$. For the small-core nano-particle, the $1 \mathrm{~nm}$ displacement did not exert a large influence on its resonant properties; displacements on the order of $3 \mathrm{~nm}$ had to be introduced to significantly alter its resonance properties. In all cases, the wavelength at which the resonance is achieved was shifted only slightly towards shorter wavelengths. The deterioration in the strength of the super-resonant states (and the associated normalized radiation resistance values) was further explained in terms of the total power spectrum and associated power flow density distributions. These results revealed that the dipole mode, which dominates the response in all cases, gets influenced more and more by an increased presence of a few additional modes (monopole and quadrupole) for the progressively larger core displacements.

We believe that the present results are useful from the experimental point of view since slight displacements of the centers of the core and shell parts of core-shell nano-particles are likely to occur in standard fabrication processes. This is more realistic for core displacements of $1 \mathrm{~nm}$. On the other hand, the larger core displacements, considered presently for the sake of completeness and the systematic nature of the study, would be those one deliberately introduces in the design in order to excite several other modes in such small nanoparticle configurations.

\section{References}

1. N.J. Halas, Nano Lett. 10 (2010) 3816-3822.
2. I. Choi, Y. Choi, IEEE J. Sel. Top. Quantum Electron. 18 (2012) 1110-1121.

3. L. Novotny, N. van Hulst, Nat. Photonics 5 (2011) 83-90.

4. A. Alú, N. Engheta, IEEE Trans. Antennas Propagat. 55 (2007) 3027-3039.

5. M.A. Noginov, G. Zhu, A.M. Belgrave, R. Bakker, V.M. Shalaev, E.E. Narimanov, S. Stout, E. Herz, T. Suteewong, U. Wiesner, Nature 460 (2009) 1110-1112.

6. J.A. Gordon, R.W. Ziolkowski, Opt. Express 15 (2007) 5.

7. N. Engheta, Science 317 (2007) 1698-1702.

8. J.A. Gordon, R.W. Ziolkowski, Opt. Express 16 (2008) 9.

9. S. Campione, M. Albani, F. Capolino, Opt. Express 1 (2011) 6.

10. I. Liberal, I. Ederra, R. Gonzalo, R.W. Ziolkowski, Phys. Rev. Appl. 1 (2014) 044002.

11. S.D. Campbell, R.W. Ziolkowski, Selected Topics in Quantum Electronics, IEEE J. Sel. Top. Quantum Electron. 19 (2013) 3.

12. J.A. Gordon, R.W. Ziolkowski, Opt. Express 15 (2007) 20.

13. J. Geng, R.W. Ziolkowski, R. Jin, X. Liang, IEEE Photonics J. 3 (2011) 6.

14. S.-Y. Liu, J. Li, F. Zhou, L. Gan, Z.-Y. Li, Opt. Express 36 (2011) 7.

15. J.-W. Liaw, C.-L. Liu, W.-M. Tu, C.-S. Sun, M.-K. Kuo, Opt. Express 18 (2010) 12.

16. S. Arslanagić, R.W. Ziolkowski, J. Opt. A 12 (2010) 024014.

17. S. Arslanagić, Y. Liu, R. Malureanu, R.W. Ziolkowski, Sensors 11 (2011) 9109-9120.

18. A.A. Kishk, R.P. Parrikar, A.Z. Elsherbeni, IEEE Trans. Antennas Propag. 40 (1992) 295-303.

19. R.Ø. Thorsen, S. Arslanagić, in Proceedings of the 9th International Congress on Advanced Electromagnetic Materials in Microwaves and Optics, Cambridge, UK, 2015.

20. R.Ø. Thorsen, S. Arslanagić, Photonics 2 (2015) 773-794.

21. J.A. Stratton, Electromagnetic Theory, McGraw-Hill, New York, 1941.

Cite this article as: Thorsen $\mathrm{R} \varnothing$ \& Arslanagic S: Effects of layer eccentricity on the super-resonant states of active cylindrical core-shell nano-particles. EPJ Appl. Metamat. 2015, $2,7$. 


\section{Appendix A: Analytical solution}

This appendix includes the details of the analytical solution pertaining to the NP configuration shown in Figure 1. The problem is solved by following the approach in [18] where plane wave scattering from an eccentric multi-layered cylinder has been considered. In here, we specialize this approach to the presently investigated core-shell NP excited by a near-by MLS. The eigenfunction expansion technique is employed in conjunction with the addition theorem for radial cylindrical functions and boundary conditions to determine the fields in the various regions.

With reference to Figure 1, the MLS field (i.e., the known incident field) in region 3 reads

$\boldsymbol{H}_{\mathrm{MLS}}=-\hat{z} I_{\mathrm{m}} \frac{\omega \varepsilon_{0}}{4}\left\{\begin{array}{l}\sum_{n=-\infty}^{\infty} H_{n}^{(2)}\left(k_{0} \rho_{s}\right) J_{n}\left(k_{0} \rho_{1}\right) e^{j n\left(\varphi_{1}-\varphi_{s}\right)} \text { for } \rho_{1} \leq \rho_{s} \\ \sum_{n=-\infty}^{\infty} H_{n}^{(2)}\left(k_{0} \rho_{1}\right) J_{n}\left(k_{0} \rho_{s}\right) e^{j n\left(\varphi_{1}-\varphi_{s}\right)} \text { for } \rho_{1} \geq \rho_{s}\end{array}\right.$

in terms of the cylindrical wave originating at $\rho_{1}$. In equation (A1), the functions $J_{n}(\cdot)$ and $H_{n}^{(2)}(\cdot)$ are the Bessel function and the Hankel function of the second kind, respectively, both of order $n$. The unknown scattered field in region 3 and the unknown total fields inside the NP are also expanded in terms of cylindrical wave functions and have the form

$$
\boldsymbol{H}_{l}=-\hat{z} I_{\mathrm{m}} \frac{\omega \varepsilon_{l}}{4} \sum_{n=-\infty}^{\infty} h_{n, l}\left(\rho_{i}\right) e^{j n \varphi_{i}}
$$

with

$$
h_{n, l}=\left\{\begin{array}{c}
B_{n}^{3} H_{n}^{(2)}\left(k_{3} \rho_{1}\right) e^{-j n \varphi_{s}}, \quad \rho_{1} \geq c \\
A_{n}^{2} J_{n}\left(k_{2} \rho_{2}\right)+B_{n}^{2} Y_{n}\left(k_{2} \rho_{2}\right), \quad a \leq \rho_{2} \leq b \\
A_{n}^{1} J_{n}\left(k_{1} \rho_{2}\right), \quad \rho_{2} \leq a
\end{array}\right.
$$

where $l$ is the region number $(l=1$, core; $l=2$, shell; and $l=3$, host), while $A_{n}^{l}$ and $B_{n}^{l}$ are the unknown expansion coefficients to be determined. The corresponding electric fields, not included in here, follow at once through the application of the Maxwell-Amperes law $\boldsymbol{E}_{l}=\nabla \times \boldsymbol{H}_{l} / j \omega \mu_{l}$. The unknown expansion coefficients are obtained upon the application of the boundary conditions requiring the continuity of the tangential components of the total electric and magnetic fields at the two interfaces of the NP. For this to be practical at the interfaces $\rho_{2}=b$ the fields in the shell region (initially expressed in terms of the $i=2$ coordinate system) must be translated to the $i=1$ coordinate system. This is accomplished through the use of the addition theorem for cylindrical wave functions of order $n$, see e.g., [21]. Thus, the application of the boundary conditions yields the following

$$
\begin{aligned}
& \varepsilon_{1} J_{n}\left(k_{1} a\right) A_{n}^{1}-\varepsilon_{2} J_{n}\left(k_{2} a\right) A_{n}^{2}-\varepsilon_{2} H_{n}^{(2)}\left(k_{2} a\right) B_{n}^{2}=0 \\
& k_{1} J_{n}^{\prime}\left(k_{1} a\right) A_{n}^{1}-k_{2} J_{n}^{\prime}\left(k_{2} a\right) A_{n}^{2}-k_{2} H_{n}^{\prime(2)}\left(k_{2} a\right) B_{n}^{2}=0
\end{aligned}
$$

$$
\begin{aligned}
& \varepsilon_{3}\left[H_{q}^{(2)}\left(k_{3} \rho_{s}\right) J_{q}\left(k_{3} b\right)+B_{q}^{3} H_{q}^{(2)}\left(k_{3} b\right)\right] e^{-j q \varphi_{s}} \\
& =\varepsilon_{2} \sum_{n=-\infty}^{\infty} J_{q-n}\left(k_{2} d_{12}\right)\left[A_{n}^{2} J_{q}\left(k_{2} b\right)+B_{n}^{2} H_{q}^{(2)}\left(k_{2} b\right)\right] e^{-j(q-n) \varphi_{12}} \\
& \varepsilon_{3}\left[H_{q}^{(2)}\left(k_{3} \rho_{s}\right) J_{q}\left(k_{3} b\right)+B_{q}^{3} H_{q}^{(2)}\left(k_{3} b\right)\right] e^{-j q \varphi_{s}} \\
& \quad=\varepsilon_{2} \sum_{n=-\infty}^{\infty} J_{q-n}\left(k_{2} d_{12}\right)\left[A_{n}^{2} J_{q}\left(k_{2} b\right)+B_{n}^{2} H_{q}^{(2)}\left(k_{2} b\right)\right] e^{-j(q-n) \varphi_{12}}
\end{aligned}
$$

at the outer interface, $\rho_{1}=b$, of the shell. The prime in the above equations denotes the derivate with respect to the entire argument of the function. In order to derive the unknown coefficients, we first use (A4a) and (A4b) to eliminate $A_{n}^{1}$. By straightforward, but lengthy calculations, one obtains

$$
\tau_{1 n} A_{n}^{2}+\tau_{2 n} B_{n}^{2}=0
$$

where

$$
\begin{gathered}
\tau_{1, n}=\frac{\varepsilon_{2}}{\varepsilon_{1}} \frac{Y_{n}\left(k_{2} a\right)}{J_{n}\left(k_{1} a\right)}-\frac{k_{2}}{k_{1}} \frac{Y_{n}^{\prime}\left(k_{2} a\right)}{J_{n}^{\prime}\left(k_{1} a\right)} \\
\tau_{2, n}=\frac{\varepsilon_{2}}{\varepsilon_{1}} \frac{J_{n}\left(k_{2} a\right)}{J_{n}\left(k_{1} a\right)}-\frac{k_{2}}{k_{1}} \frac{J_{n}^{\prime}\left(k_{2} a\right)}{J_{n}^{\prime}\left(k_{1} a\right)} .
\end{gathered}
$$

The result in (A6) can be written in the following matrix form

$$
\left[\begin{array}{ll}
\boldsymbol{\tau}_{1, n} & \tau_{2, n}
\end{array}\right]\left[\begin{array}{l}
\boldsymbol{A}_{n}^{2} \\
\boldsymbol{B}_{n}^{2}
\end{array}\right]=[\mathbf{0}]
$$

where $\tau_{i, n}(i=1,2)$ is a diagonal matrix, $\boldsymbol{A}_{n}^{2}$ and $\boldsymbol{B}_{n}^{2}$ are vectors containing the expansion coefficients for all $n$, and $\mathbf{0}$ denotes the zero vector. In a more explicit form, the diagonal matrix $\tau_{i, n}$ reads

$$
\tau_{i, n}=\left[\begin{array}{ccccc}
\tau_{i,-N} & 0 & \cdots & 0 & 0 \\
0 & \tau_{i,-N+1} & \cdots & 0 & 0 \\
\vdots & \vdots & \ddots & \vdots & \vdots \\
0 & 0 & \cdots & \tau_{i,-N-1} & 0 \\
0 & 0 & \cdots & 0 & \tau_{i, N}
\end{array}\right]
$$

where $N$ is the truncation limit of the infinite expansions in (A1)-(A3).

Consider next the outer interface, $\rho_{1}=b$, of the shell. The results in (A5a) and (A5b) are used to eliminate $B_{n}^{3}$, and by tedious manipulations, the resulting expression is simplified to yield

$$
\sum_{n=-N}^{N}\left[\psi_{1, q, n} A_{n}^{2}+\psi_{2, q, n} B_{n}^{2}\right]=\chi_{q}
$$


where

$\psi_{1, q, n}=J_{q-n}\left(k_{2} d_{12}\right)\left(\frac{\varepsilon_{2}}{\varepsilon_{3}} \frac{J_{q}\left(k_{2} b\right)}{H_{q}^{(2)}\left(k_{3} b\right)}-\frac{k_{2}}{k_{3}} \frac{J_{q}^{\prime}\left(k_{2} b\right)}{H_{q}^{\prime(2)}\left(k_{3} b\right)}\right) e^{-j(q-n) \varphi_{12}+j q \varphi_{s}}$

(A11a)

$\psi_{2, q, n}=J_{q-n}\left(k_{2} d_{12}\right)\left(\frac{\varepsilon_{2}}{\varepsilon_{3}} \frac{Y_{q}\left(k_{2} b\right)}{H_{q}^{(2)}\left(k_{3} b\right)}-\frac{k_{2}}{k_{3}} \frac{Y_{q}^{\prime}\left(k_{2} b\right)}{H_{q}^{\prime(2)}\left(k_{3} b\right)}\right) e^{-j(q-n) \varphi_{12}+j q \varphi_{s}}$

$$
\chi_{q}=H_{q}^{(2)}\left(k_{3} \rho_{s}\right)\left(\frac{J_{q}\left(k_{3} b\right)}{H_{q}^{(2)}\left(k_{3} b\right)}-\frac{J_{q}^{\prime}\left(k_{3} b\right)}{H_{q}^{\prime(2)}\left(k_{3} b\right)}\right)
$$
face is

The matrix form of (A8) for the boundary at the outer inter-

$$
\left[\begin{array}{ll}
\boldsymbol{\psi}_{1, q, n} & \boldsymbol{\psi}_{2, q, n}
\end{array}\right]\left[\begin{array}{l}
\boldsymbol{A}_{\boldsymbol{n}}^{2} \\
\boldsymbol{B}_{n}^{2}
\end{array}\right]=\left[\boldsymbol{\chi}_{q}\right]
$$

where $\psi_{i, q, n}$ is a matrix, and $\chi_{q}$ a vector. In a more explicit form,

$$
\psi_{i, q, n}=\left[\begin{array}{ccccc}
\psi_{i,-N,-N} & \psi_{i,-N,-N+1} & \cdots & \psi_{i,-N, N-1} & \psi_{i,-N, N} \\
\psi_{i,-N+1,-N} & \psi_{i,-N+1,-N} & \cdots & \psi_{i,-N+1, N-1} & \psi_{i,-N+1, N} \\
\vdots & \vdots & \ddots & \vdots & \vdots \\
\psi_{i, N-1,-N} & \psi_{i, N-1,-N+1} & \cdots & \psi_{i,-N+1, N-1} & \psi_{i, N-1, N} \\
\psi_{i, N,-N} & \psi_{i, N,-N+1} & \cdots & \psi_{i, N, N-1} & \psi_{i, N, N}
\end{array}\right]
$$

To implement the solution of these relations, it is convenient to write (A8) and (A12) as

$$
\left[\begin{array}{cc}
\boldsymbol{\psi}_{1, q, n} & \boldsymbol{\psi}_{2, q, n} \\
\boldsymbol{\tau}_{1, n} & \boldsymbol{\tau}_{2, n}
\end{array}\right]\left[\begin{array}{l}
\boldsymbol{A}_{n}^{2} \\
\boldsymbol{B}_{n}^{2}
\end{array}\right]=\left[\begin{array}{c}
\boldsymbol{\chi}_{q} \\
\mathbf{0}
\end{array}\right]
$$

or, by use of (A9) and (A13), in the explicit form as

$$
\left[\begin{array}{cccccc}
\psi_{1,-N,-N} & \cdots & \psi_{1,-N, N} & \psi_{2,-N,-N} & \cdots & \psi_{2,-N, N} \\
\vdots & \ddots & \vdots & \vdots & \ddots & \vdots \\
\psi_{1, N,-N} & \cdots & \psi_{1, N, N} & \psi_{2, N,-N} & \cdots & \psi_{2, N, N} \\
\tau_{1,-N} & 0 & 0 & \tau_{2,-N} & 0 & 0 \\
0 & \ddots & 0 & 0 & \ddots & 0 \\
0 & 0 & \tau_{1, N} & 0 & 0 & \tau_{2, N}
\end{array}\right]\left[\begin{array}{c}
A_{-N}^{2} \\
\vdots \\
A_{N}^{2} \\
B_{-N}^{2} \\
\vdots \\
B_{N}^{2}
\end{array}\right]=\left[\begin{array}{c}
\chi_{-N} \\
\vdots \\
\chi_{N} \\
0 \\
\vdots \\
0
\end{array}\right]
$$

A more compact notation for the result in (A15) takes the form of

$$
\boldsymbol{M}_{n} \boldsymbol{\Psi}_{n}=\boldsymbol{\Lambda}_{n}
$$

where $\boldsymbol{M}_{n}$ is the matrix containing the information about the cylindrical waves at the interfaces, $\boldsymbol{\Psi}_{n}$ is the vector containing the unknown expansion coefficients of the fields in region 2, while $\boldsymbol{\Lambda}_{n}$ is the excitation vector, which depends on the location of the MLS, as well as on the value of the cylindrical waves at the outer interface. The solution to (A16a) is given by

$$
\boldsymbol{\Psi}_{n}=\left[\boldsymbol{M}_{n}\right]^{-1} \boldsymbol{\Lambda}_{n}
$$

thereby determining the unknown expansion coefficients in the shell region. When these are known, the expansion coefficients in the core and outside the NP are obtained by solving (A4a) and (A5a), respectively, with the following result

$$
A_{n}^{1}=\frac{\varepsilon_{2}}{\varepsilon_{1}} \frac{J_{n}\left(k_{3} a\right) A_{n}^{2}+Y_{n}^{(2)}\left(k_{2} a\right) B_{n}^{2}}{J_{n}\left(k_{1} a\right)}
$$

$$
\begin{aligned}
B_{n}^{3}=\left[\frac{\varepsilon_{2}}{\varepsilon_{3}} \sum_{n=-\infty}^{\infty}\{\right. & \left.J_{q-n}\left(k_{2} d_{12}\right)\left[J_{q}\left(k_{2} b\right) A_{n}^{2}+Y_{q}\left(k_{2} b\right) B_{n}^{2}\right] e^{-j(q-n) \varphi_{12}+j q \varphi_{s}}\right\} \\
& \left.-H_{q}^{(2)}\left(k_{3} \rho_{s}\right) J_{q}\left(k_{3} a\right)\right] \frac{1}{H_{q}^{(2)}\left(k_{3} b\right)} \quad(\mathrm{A} 17 \mathrm{~b})
\end{aligned}
$$

\section{Appendix B: Comparison of analytical and numerical representative results}

The analytical results and their implementation were extensively validated by several means. First, the code developed in Matlab to treat the eccentrically layered NP in Figure 1 was used to reproduce successfully the known results for the corresponding concentric NPs considered previously in [17]. Moreover, the NP configuration in Figure 1 was analyzed numerically in Comsol Multiphysics to further validate the correctness of the developed analytical code. A few representative results from Comsol Multiphysics are compared below with our analytical results and demonstrate a perfect agreement between the two methods of analysis.

Figure A1 shows the NRR as a function of the wavelength $\lambda$ for the small-core (a) and large-core (b) NP. In both cases, the core is displaced along the positive $x$-axis by an amount equal to $x_{12}=5 \mathrm{~nm}$. A perfect agreement is found between the analytical and numerical results throughout the investigated wavelength range. This comparison further confirms the correctness of the analytical results and the developed code.

The magnetic fields corresponding to the NRR maxima in Figure A1 are shown in Figure A2. More specifically, the quantity $20 \log _{10}|\boldsymbol{H}|[\mathrm{dB}]$, with $\boldsymbol{H}$ being the total magnetic field normalized by $1(\mathrm{~A} / \mathrm{m})$ prior to taking the logarithm, is shown in a square region of $90 \times 90 \mathrm{~nm}^{2}$. Curves illustrating the cylindrical surfaces of the NP are also shown. Figure A2 shows the field for the small-core case as obtained by the analytical solution (a) and numerical solution (b); the corresponding largecore case is shown in Figure A2c and Figure A2d. For both core cases, an excellent agreement is found between the analytical and numerical results. 


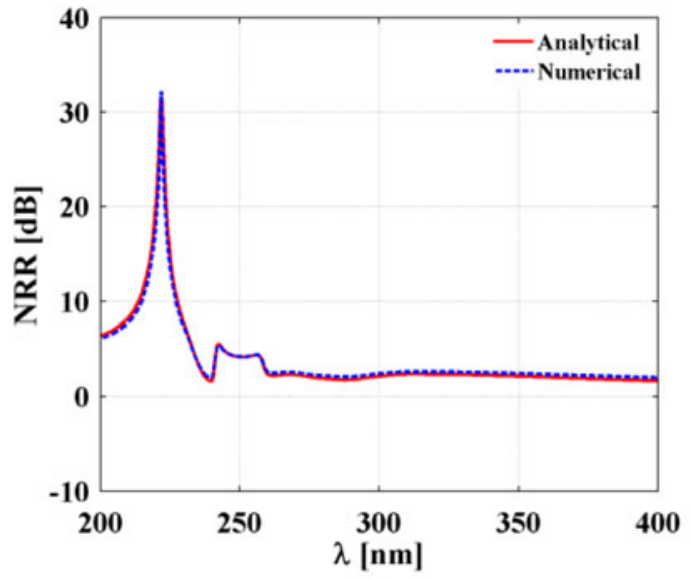

(a)

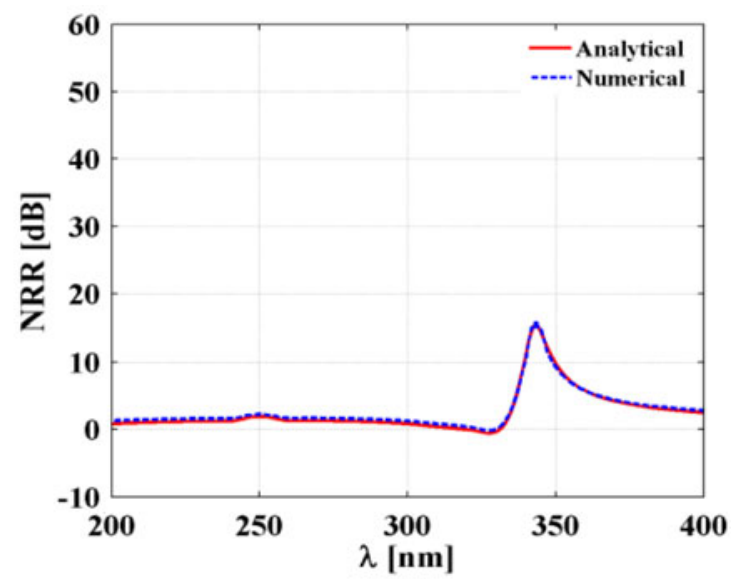

(b)

Figure A1. NRR as a function of the wavelength $(\lambda)$ for the small-core (a) and large-core (b) NP. In both cases, the core is displaced along the positive $x$-axis by an amount of $x_{12}=5 \mathrm{~nm}$. Analytical (Matlab) and numerical (Comsol Multiphysics) results are compared.

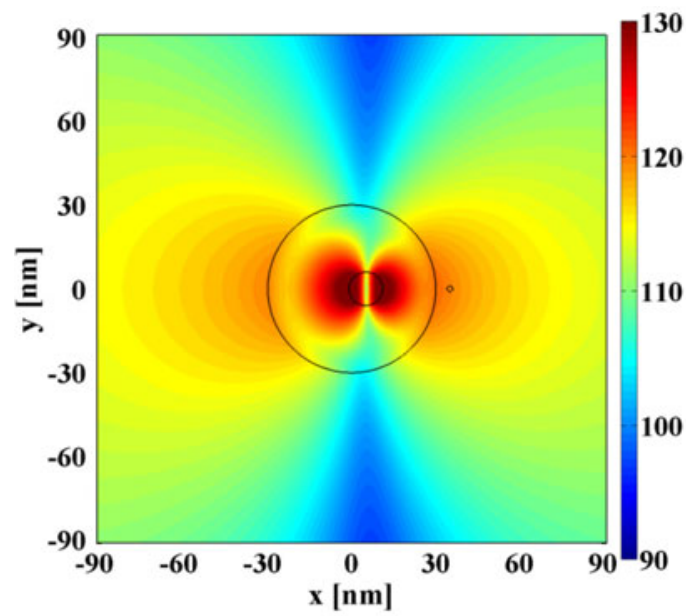

(a)

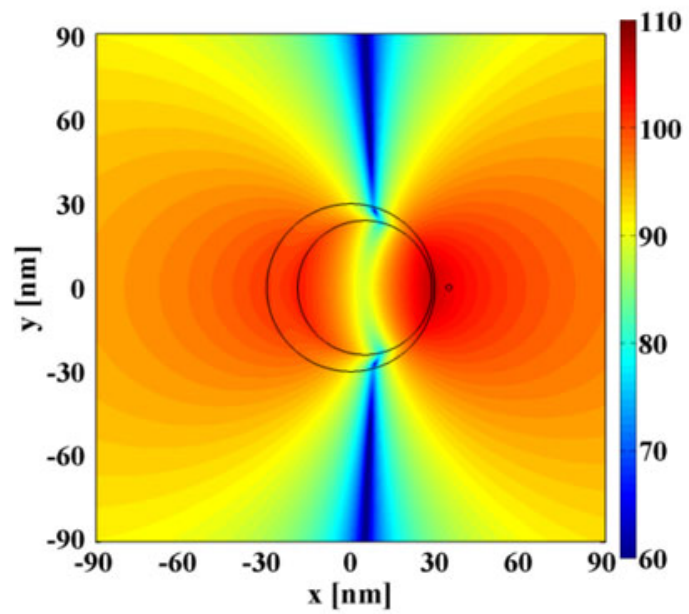

(c)

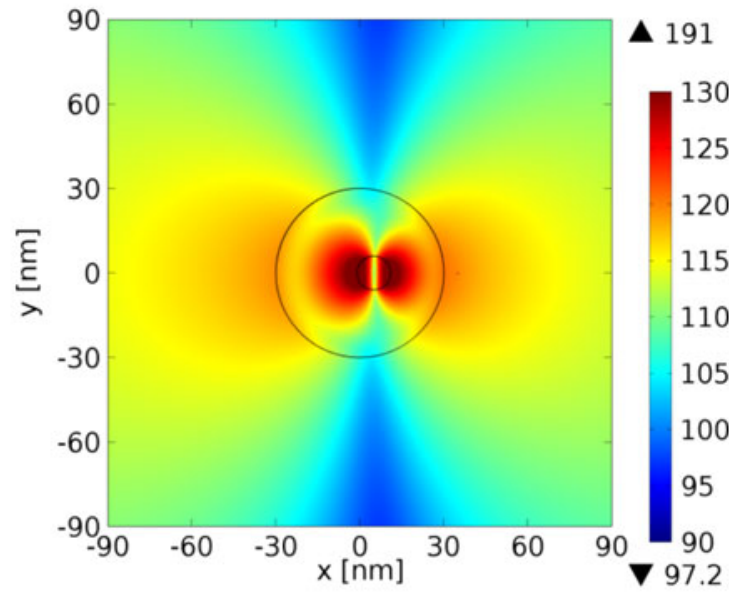

(b)

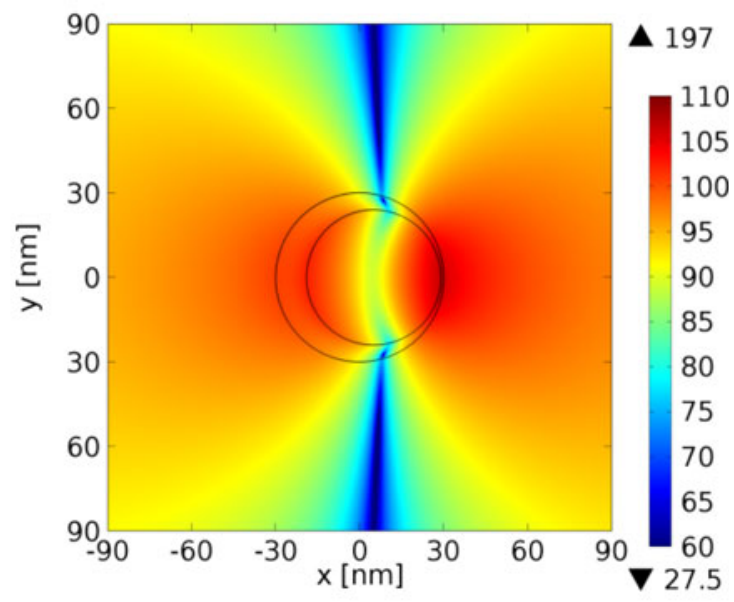

(d)

Figure A2. The magnitude of the magnetic field, $20 \log _{10}|\boldsymbol{H}|$, for the small-core NP (a) and (b), and the large-core NP (c) and (d). In both cases, the cores displaced along the positive $x$-axis by an amount equal to $x_{12}=5 \mathrm{~nm}$. Analytical (Matlab) results are shown in (a) and (c), while numerical (Comsol Multiphysics) results are shown in (b) and (d). 